\title{
Analytical Evaluation of the Performance of Proportional Fair Scheduling in OFDMA-Based Wireless Systems
}

\author{
Mohamed H. Ahmed, Octavia A. Dobre, and Rabie K. Almatarneh \\ Faculty of Engineering and Applied Science, Memorial University, St. John's, NL, Canada A1B 3X5 \\ Correspondence should be addressed to Mohamed H. Ahmed, mhahmed@mun.ca
}

Received 2 March 2012; Accepted 7 May 2012

Academic Editor: Yi Su

Copyright (c) 2012 Mohamed H. Ahmed et al. This is an open access article distributed under the Creative Commons Attribution License, which permits unrestricted use, distribution, and reproduction in any medium, provided the original work is properly cited.

This paper provides an analytical evaluation of the performance of proportional fair (PF) scheduling in Orthogonal FrequencyDivision Multiple Access (OFDMA) wireless systems. OFDMA represents a promising multiple access scheme for transmission over wireless channels, as it combines the orthogonal frequency division multiplexing (OFDM) modulation and subcarrier allocation. On the other hand, the PF scheduling is an efficient resource allocation scheme with good fairness characteristics. Consequently, OFDMA with PF scheduling represents an attractive solution to deliver high data rate services to multiple users simultaneously with a high degree of fairness. We investigate a two-dimensional (time slot and frequency subcarrier) PF scheduling algorithm for OFDMA systems and evaluate its performance analytically and by simulations. We derive approximate closedform expressions for the average throughput, throughput fairness index, and packet delay. Computer simulations are used for verification. The analytical results agree well with the results from simulations, which show the good accuracy of the analytical expressions.

\section{Introduction}

OFDMA is a promising solution for the high data-rate coverage required in multiuser broadband wireless communications. Current and evolving standards for broadband wireless systems, such as IEEE 802.16e, have proposed OFDMA as the multiple access technique for the air interface. OFDMA is a multiple access technique which is based on OFDM. In OFDM systems, a single user gets access to the whole available spectrum at any time instant, and, as a result, multiple users share resources using time scheduling. On the other hand, in OFDMA systems users share the available spectrum using subcarrier allocation. Hence, OFDMA requires scheduling in both time and frequency domains (time slots and frequency subcarriers). This additional degree of freedom makes the scheduling problem in OFDMA systems more challenging, but also more effective.

Scheduling plays a key role in the OFDMA systems resource management [1]. Efficient scheduling implies effective utilization of the available radio resources, high throughput, low packet delay, and fair treatment of all users in the system. Various scheduling techniques have been proposed for OFDMA systems [1-4]. For example, a maximum carrier-to-interference ratio-based scheduling algorithm is adopted in [1] to provide a more fair treatment among users, while in [2] the resource allocation problem is studied with and without service request constraints. Two-dimensional matrix-based scheduling algorithms are proposed in [2] using the raster scanning approach to achieve high system throughput with relatively lower complexity.

The PF algorithm is an appealing scheduling scheme to meet the quality of service requirements in OFDMA systems [5-8], as it can improve the fairness among users without sacrificing the efficiency in terms of average (or aggregate) throughput. With this algorithm, the level of satisfaction and starvation of all users in the system is sensed over time, and resources are assigned to users based on that. Moreover, the PF algorithm is flexible and can 
scale between fairness and efficiency. In [8], we propose an iterative two-dimensional (time symbols and frequency subbands) PF scheduling for OFDMA systems. However, the performance of PF scheduling for OFDMA systems is not determined analytically and it is usually determined by computer simulations.

An analytical method, which is based on the Gaussian approximation of the instantaneous data rate in a Rayleigh fading environment, is used to analyze the performance of PF scheduling in [9]. However, this method is developed for single-carrier systems and limited to the case of users with full buffers. We adopt the methodology in [9] to develop an analytical solution for the PF scheduling in OFDMA systems for bursty traffic conditions and full buffers scenario, as well. In this paper, we provide approximate closed-form expressions for the average throughput and throughput fairness index of our PF scheduling scheme proposed for OFDMA systems in [8]. In addition, simulation results are provided in the paper to check the accuracy of the analytical method.

The rest of this paper is organized as follows: Section 2 describes the OFDMA system model. The PF scheduling algorithm is provided in Section 3. The closed-form analytical derivations of the throughput, fairness index, and delay are presented in Section 4. Then, Section 5 provides numerical results from the analytical solution, as well as simulation outcomes. Finally, conclusions are provided in Section 6.

\section{System Model}

As shown in Figure 1, the OFDMA system resources have two dimensions: frequency and time. In frequency domain, the signal bandwidth is divided into a plurality of subbands, which contain highly correlated orthogonal subcarriers. A number of $S$ subcarriers are grouped into $M$ subbands, each with $K=S / M$ subcarriers. In time domain, data is organized in frames, which are further divided in time symbols. The minimum allocable resource unit in the system is defined by the intersection between a subband in frequency domain and time symbol in time domain.

We consider a single-cell scenario, with $N$ users with bursty traffic demands. The signals are affected by path loss, lognormal shadowing, and Rayleigh fading. The smallest data entity which the base station can handle is a fixedsize data packet. We use the Poisson traffic model. The cell shape is circular and the base station is located at the center. Users are uniformly distributed over the cell area. We consider the downlink only. However, the analysis can be easily extended to the uplink case. Moreover, adaptive coding and modulation (ACM) is used to enhance the resource utilization. The suitable modulation level and coding rate are decided depending on the channel state information (CSI) for each subband. Table 1 shows the ACM schemes used in this paper, along with the corresponding signal-to-noise ratios (SNRs).

The frequency subcarriers are correlated in the frequency domain. The fading affecting the frequency subcarriers has

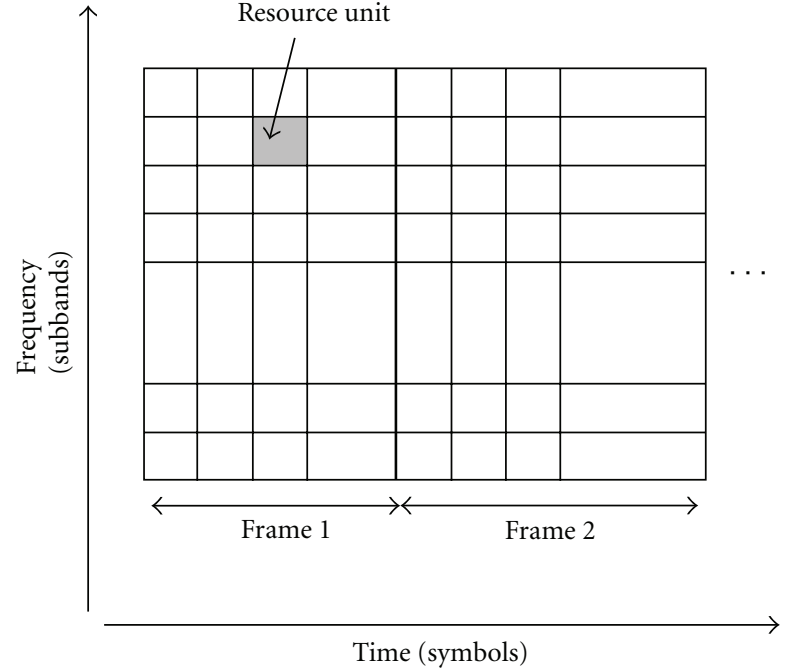

Figure 1: Two-dimensional resources in OFDMA systems.

TABLE 1: Adaptive coding and modulation parameters.

\begin{tabular}{lccc}
\hline Modulation format & Code rate & Bits/symbol & SNR $(\mathrm{dB})$ \\
\hline BPSK & $1 / 4$ & $1 / 4$ & -2.9 \\
BPSK & $1 / 2$ & $1 / 2$ & -0.2 \\
QPSK & $1 / 2$ & 1 & 2.2 \\
8PSK & $1 / 2$ & $3 / 2$ & 5.2 \\
8PSK & $2 / 3$ & 2 & 8.4 \\
64QAM & $1 / 2$ & 3 & 11.8 \\
64QAM & $2 / 3$ & 4 & 15.1 \\
\hline
\end{tabular}

cross correlation because of the coherence bandwidth of the wireless channel [10]. A frequency selective Rayleigh fading channel is modeled based on [10-12]. The frequency selective Rayleigh subcarriers are generated with correlation between them in the frequency domain, where the complex valued correlation is formulated as a function of frequency separation between the subcarriers. In order to minimize the bit error rate and improve the OFDMA system reliability, we consider the worst case subcarrier fading in each subband for the SNR and link budget calculations. Although the worst case subcarrier fading is considered in a subband while selecting an ACM scheme, the overall SNR calculation does not significantly change because the fading difference between subcarriers within a subband is insignificant because the fading coefficients are highly correlated.

\section{PF Scheduling Algorithm for OFDMA Systems}

Closed-form expressions are subsequently derived for the throughput and fairness index for the PF scheduling algorithm that we proposed in [8]. The algorithm is briefly explained, followed by its analytical performance analysis. 
According to the PF scheduling algorithm that we develop in [8] for OFDMA systems, the user with the index

$$
k=\arg \max _{1 \leq i \leq N} \frac{D_{i j}(n)}{R_{i}(n-1)},
$$

is ranked first among the $N$ users on subband $j, j=1, \ldots, M$. Here, $D_{i j}(n)$ is the instantaneous data rate of user $i, i=$ $1, \ldots, N$ on subband $j$ at time frame $n$, and $R_{i \backslash}(n)$ is the timeaverage data rate of user $i$ at time frame $n$. The time-average data rate is updated at the end of a time frame for each user $i$ on all the available subbands as follows:

$$
R_{i}(n)= \begin{cases}\left(1-T_{c}^{-1}\right) R_{i}(n-1), & i \neq k, \\ \left(1-T_{c}^{-1}\right) R_{i}(n-1)+T_{c}^{-1} \sum_{\substack{j=1, j \in S_{i}(n)}}^{M} D_{i j}(n), & i=k,\end{cases}
$$

where $S_{i}(n)$ represents the set of subbands assigned to user $i$ during time frame $n$, and $T_{c}$ is the averaging window expressed in time frames which controls the amount of historical information taken into account when sharing the resources among multiple users and can be chosen to achieve a desirable throughput-fairness tradeoff. User $i$ is scheduled on time frame $n$ if $i=k$ and is not scheduled if $i \neq k$.

Since the packet arrival is assumed to be bursty, the best user (chosen by (1)) might have empty buffer. In this case, the subband assigned to the best user should be given to the second best user if this has nonempty buffer. If not, the subband is assigned to the third best users and so on, where the ranking of users is based on the same criterion used in (1), that is, $D_{i j}(n) / R_{i j}(n-1)$. As such, we modify (2) as follows:

$$
\begin{aligned}
R_{i}(n)= & \left(1-T_{c}^{-1}\right) R_{i}(n-1) \alpha T_{c}^{-1} \sum_{j=1}^{M} I_{i j}^{1}(n) D_{i j}(n) \\
& +\alpha(1-\alpha) T_{c}^{-1} \sum_{j=1}^{M} I_{i j}^{2}(n) D_{i j}(n) \\
& +\alpha(1-\alpha)^{2} T_{c}^{-1} \sum_{j=1}^{M} I_{i j}^{3}(n) D_{i j}(n) \\
& +\cdots+\alpha(1-\alpha)^{N-1} T_{c}^{-1} \sum_{j=1}^{M} I_{i j}^{N}(n) D_{i j}(n) \\
= & \left(1-T_{c}^{-1}\right) R_{i}(n-1)+\alpha T_{c}^{-1} \sum_{k=1}^{N}(1-\alpha)^{k-1} \\
& \times \sum_{j=1}^{M} I_{i j}^{k}(n) D_{i j}(n),
\end{aligned}
$$

where $I_{i j}^{k}(n), k=1, \ldots, N$, represents a selector indicator which equals 1 if user $i$ is ranked $k$ th on subband $j$ and frame $n$ and equals 0 otherwise, and $\alpha$ is the probability that the buffer of user $i$ is not empty. We assume that $\alpha$ is the same for all users. The terms in the right-hand side of (3) represent the potential achievable throughput for a user. The first term reflects the average throughput achieved by the round-robin (RR) algorithm, while the remaining $N$ terms represent the additional average throughput provided by our algorithm when compared with RR. The first term (out of the remaining $N$ terms) represents the additional average throughput when user $i$ is ranked first and assigned subband $j$. The second term (out of the remaining $N$ terms) reflects the additional average throughput when user $i$ is ranked second and assigned subband $j$ because the user ranked first has empty buffer, and so on.

The PF scheduling algorithm consists of two steps [8]. In the first step, all users in the system are ranked. A resource matrix that contains the ranking of all users on all subbands is generated based on (1). The instantaneous data rate, $D_{i j}(n)$, represents the efficiency factor, whereas the historical average rate combined with $T_{c}$ represents the fairness factor. As such, the ranking of the users reflects both the channel gain and shortage of service. In the second step, scheduling is performed based on the ranking and demands of the users on one hand and the resource accessibility on the other hand. The algorithm iteratively serves the user with the highest rank among all users on all subbands.

A user will be excluded from the waiting users' list if all waiting packets are served. This algorithm allows subband sharing in time domain, where different time symbols in the subband can be utilized by different users. A subband will be eliminated from the resource matrix if the remaining resources cannot support at least one packet for any requesting user within this time frame. The algorithm tracks the satisfaction levels of all users at the end of each time frame by updating the historical data rate, $R_{i}(n)$, using (2).

\section{Performance Analysis}

4.1. Average Throughput. It is shown that assuming a linear relationship between the instantaneous data rate, $D_{i j}(n)$, and the SNR is unrealistic under Rayleigh fading environment $[9,13]$. Actually, it is demonstrated that it is more realistic to assume that $D_{i j}(n)$ follows a Gaussian distribution with mean and variance given, respectively, as follows [9]:

$$
\begin{aligned}
E\left[D_{i j}\right]= & \int_{0}^{\infty} \log \left(1+\mathrm{SNR}_{i j} \gamma\right) e^{-\gamma} d \gamma \\
\sigma_{D_{i j}}^{2}= & \int_{0}^{\infty} \log \left(1+\operatorname{SNR}_{i j} \gamma\right)^{2} e^{-\gamma} d \gamma \\
& -\left(\int_{0}^{\infty} \log \left(1+\operatorname{SNR}_{i j} \gamma\right) e^{-\gamma} d \gamma\right)^{2},
\end{aligned}
$$

where $E[\cdot]$ denotes the expectation operator. According to the PF algorithm presented in (1) and (2), one can express 
the average achievable throughput of user $i$ on all the available subbands in the time frame $n$ as follows:

$$
\begin{aligned}
E\left[R_{i}(n)\right]= & \left(1-T_{c}^{-1}\right) E\left[R_{i}(n-1)\right] \\
& +\alpha T_{c}^{-1} \sum_{k=1}^{N}(1-\alpha)^{k-1} E\left[\sum_{j=1}^{M} I_{i j}^{k}(n) D_{i j}(n)\right] .
\end{aligned}
$$

We can rewrite (5) as follows:

$$
\begin{aligned}
E\left[R_{i}(n)\right]= & \left(1-T_{c}^{-1}\right) E\left[R_{i}(n-1)\right] \\
& +\alpha T_{c}^{-1} \sum_{k=1}^{N}(1-\alpha)^{k-1} \\
& \times E\left[\sum_{j=1}^{M} D_{i j}(n) \mid I_{i j}^{k}(n)=1\right] \operatorname{Pr}\left(I_{i j}^{k}(n)=1\right),
\end{aligned}
$$

where $\operatorname{Pr}\left(I_{i j}^{k}(n)=1\right)$ is the probability that user $i$ is ranked $k$ th on subband $j$ and time frame $n$. Under the assumption of stationary throughput [9], $R_{i}$, and independent subbands, one can further express (6) as follows:

$$
\begin{aligned}
E\left[R_{i}\right]= & \alpha \sum_{k=1}^{N}(1-\alpha)^{k-1} \\
& \times E\left[\sum_{j=1}^{M} D_{i j}(n) \mid I_{i j}^{k}(n)=1\right] \operatorname{Pr}\left(I_{i j}^{k}(n)=1\right) .
\end{aligned}
$$

By applying the Bayes' theorem, (7) can be rewritten as follows:

$$
\begin{aligned}
E\left[R_{i}\right]= & \alpha \sum_{k=1}^{N}(1-\alpha)^{k-1} \\
& \times \sum_{j=1}^{M} \int_{-\infty}^{\infty} x f_{D_{i j}}(x) \operatorname{Pr}\left(I_{i j}^{k}(n)=1 \mid D_{i j}(n)=x\right) d x,
\end{aligned}
$$

where $f_{D_{i j}}(\cdot)$ denotes the probability density function (pdf) of $D_{i j}$. By assuming independent $D_{i j}$ and based on the PF selection criterion presented in (1), we can determine the conditional ranking probabilities as follows:

$$
\begin{aligned}
& \operatorname{Pr}\left(I_{i j}^{1}(n)=1 \mid D_{i j}(n)=x\right)=\prod_{\substack{l=1 \\
l \neq i}}^{N} F_{D_{l j}}\left(x \frac{R_{l}(n)}{R_{i}(n)}\right), \\
& \operatorname{Pr}\left(I_{i j}^{2}(n)=1 \mid D_{i j}(n)=x\right) \\
& =\left(1-F_{D_{l_{1} j}}\left(x \frac{R_{l_{1}}(n)}{R_{i}(n)}\right)\right) \prod_{\substack{l=1 \\
l \neq i, l_{1}}}^{N} F_{D_{l j}}\left(x \frac{R_{l}(n)}{R_{i}(n)}\right),
\end{aligned}
$$

$$
\begin{gathered}
\operatorname{Pr}\left(I_{i j}^{3}(n)=1 \mid D_{i j}(n)=x\right) \\
=\left(1-F_{D_{l_{1} j}}\left(x \frac{R_{l_{1}}(n)}{R_{i}(n)}\right)\right)\left(1-F_{D_{l_{2} j}}\left(x \frac{R_{l_{2}}(n)}{R_{i}(n)}\right)\right) \\
\times \prod_{\substack{l=1 \\
l \neq i, l_{1}, l_{2}}}^{N} F_{D_{l j}}\left(x \frac{R_{l}(n)}{R_{i}(n)}\right), \\
\vdots \\
\operatorname{Pr}\left(I_{i j}^{N}(n)=1 \mid D_{i j}(n)=x\right) \\
=\prod_{\substack{l=1 \\
l \neq i}}^{N}\left(1-F_{D_{l j}}\left(x \frac{R_{l}(n)}{R_{i}(n)}\right)\right)
\end{gathered}
$$

where $F_{D_{i j}}(\cdot)$ is the cumulative distribution function (cdf) of $D_{i j}$, while $l_{1}$ and $l_{2}$ are the indexes of the users ranked the first and the second (on subband $j$ ), respectively. By using (9) and the Gaussian pdf of $D_{i j}$, and under the assumptions that $T_{c} \rightarrow \infty$ and $R_{i}$ is an ergodic process (such that its moving average equals the statistical average), now (9) can be re-written as follows:

$$
\begin{aligned}
& \operatorname{Pr}\left(I_{i j}^{1}(n)=1 \mid D_{i j}(n)=x\right) \approx \prod_{\substack{l=1 \\
l \neq i}}^{N} F_{D_{l j}(n)}\left(\frac{E\left[R_{l}\right]}{E\left[R_{i}\right]} x\right) \\
& \operatorname{Pr}\left(I_{i j}^{2}(n)=1 \mid D_{i j}(n)=x\right) \\
& \approx\left(1-F_{D_{i j}(n)}\left(\frac{E\left[R_{l}\right]}{E\left[R_{i}\right]} x\right)\right) \\
& \times \prod_{\substack{l=1 \\
l \neq i}}^{N-1} F_{D_{l j}(n)}\left(\frac{E\left[R_{l}\right]}{E\left[R_{i}\right]} x\right) \\
& \operatorname{Pr}\left(I_{i j}^{3}(n)=1 \mid D_{i j}(n)=x\right) \\
& \approx\left(1-F_{D_{i j}(n)}\left(\frac{E\left[R_{l}\right]}{E\left[R_{i}\right]} x\right)\right) \prod_{\substack{l=1 \\
l \neq i}}^{2 N-2} F_{D_{l j}(n)}\left(\frac{E\left[R_{l}\right]}{E\left[R_{i}\right]} x\right), \\
& \operatorname{Pr}\left(I_{i j}^{N}(n)=1 \mid D_{i j}(n)=x\right)=\prod_{\substack{l=1 \\
l \neq i}}^{N}\left(1-F_{D_{l j}}\left(\frac{E\left[R_{l}\right]}{E\left[R_{i}\right]} x\right)\right) .
\end{aligned}
$$


Hence, (8) can be expressed as follows:

$$
\begin{aligned}
E\left[R_{i}\right]= & \alpha \sum_{k=1}^{N}(1-\alpha)^{k-1} \\
& \times \sum_{j=1}^{M}\left[\int_{-\infty}^{\infty} x f_{D_{i j}(n)}(x)\left[1-F_{R_{i j}(n)}\left(\frac{E\left[R_{l}\right]}{E\left[R_{i}\right]} x\right)\right]^{k-1}\right. \\
& \left.\times \prod_{\substack{l=1 \\
l \neq i}}^{N-k+1} F_{D_{l j}(n)}\left(\frac{E\left[R_{l}\right]}{E\left[R_{i}\right]}\right) d x\right] .
\end{aligned}
$$

By assuming a Gaussian distribution of the instantaneous traffic rate, (11) becomes

$$
\begin{aligned}
E\left[R_{i}\right]=\alpha \sum_{k=1}^{N} & (1-\alpha)^{k-1} \\
& \times \sum_{j=1}^{M}\left[\int_{-\infty}^{\infty}\left(y \sigma_{D_{i j}}+E\left[D_{i j}\right]\right) \frac{e^{-y^{2} / 2}}{\sqrt{2 \pi}}\right. \\
& \times\left[1-F_{R_{i j}(n)}\left(\frac{E\left[R_{l}\right]}{E\left[R_{i}\right]}\left(y \sigma_{D_{i j}}+E\left[D_{i j}\right]\right)\right)\right]^{k-1} \\
& \left.\times \prod_{\substack{l=1, l \neq i}}^{N-k+1} F_{D_{l j}(n)}\left(\frac{E\left[R_{l}\right]}{E\left[R_{i}\right]}\left(y \sigma_{D_{i j}}+E\left[D_{i j}\right]\right)\right) d y\right] .
\end{aligned}
$$

Now, assume $\left.E\left[R_{l}\right] / E\left[R_{i}\right]\right)=\left(E\left[D_{l}\right] / E\left[D_{i}\right]\right.$, so, $F_{R_{i j}(n)}\left(y \sigma_{D_{i j}}+E\left[D_{i j}\right]\right)$ can be re-written as $[8]$

$$
F_{R_{i j}(n)}\left(\frac{E\left[R_{l}\right]}{E\left[R_{i}\right]}\left(y \sigma_{D_{i j}}+E\left[D_{i j}\right]\right)\right)=F_{(0,1)}\left(\frac{E\left[D_{l i}\right] \sigma_{D_{l i}}}{E\left[R_{i j}\right] \sigma_{D_{i j}}} y\right)
$$

where $F_{(0,1)}(\cdot)$ represents the standard normal cdf with zero-mean and unit-variance. Furthermore, we assume a proportional relationship between the mean and standard deviation of all users in the system [8]; hence, the previous expression can be approximated as

$$
F_{(0,1)}\left(\frac{E\left[D_{l i}\right] \sigma_{D_{l i}}}{E\left[R_{i j}\right] \sigma_{D_{i j}}} y\right)=F_{(0,1)}(y)
$$

After some mathematical manipulations, one can further express (12) as

$$
\begin{aligned}
E\left[R_{i}\right]= & \alpha \sum_{k=1}^{N}(1-\alpha)^{k-1} \\
& \times \sum_{j=1}^{M}\left[\sigma_{D_{i j}} \int_{-\infty}^{\infty} y \frac{e^{-y^{2} / 2}}{\sqrt{2 \pi}}\left(1-F_{(0,1)}(y)\right)^{k-1} F_{(0,1)}^{N-k}(y) d y\right. \\
& \left.+E\left[D_{\mathrm{ij}}\right] \int_{0}^{1}\left(1-F_{(0,1)}(y)\right)^{k-1} F_{(0,1)}^{N-k}(y) d F_{(0,1)}(y)\right] .
\end{aligned}
$$

It is straightforward to show that

$$
\int_{0}^{1} F_{(0,1)}^{N-1}(y) d F_{(0,1)}(y)=\frac{1}{N} .
$$

Then, one can easily find that

$$
\int_{0}^{1}\left(1-F_{(0,1)}(y)\right) F_{(0,1)}^{N-2}(y) d F_{(0,1)}(y)=\frac{1}{N(N-1)},
$$

and, finally, through the mathematical induction, we can write

$$
\begin{gathered}
\int_{0}^{1}\left(1-F_{(0,1)}(y)\right)^{k-1} F_{(0,1)}^{N-k}(y) d F_{(0,1)}(y) \\
=\frac{(k-1) !(N-k) !}{N !}, \quad k=1, \ldots, N .
\end{gathered}
$$

Thus, (15) can be expressed as follows:

$$
\begin{aligned}
E\left[R_{i}\right]= & \alpha \sum_{k=1}^{N}(1-\alpha)^{k-1} \\
& \times \sum_{j=1}^{M} \sigma_{D_{i j}} \int_{-\infty}^{\infty} y \frac{e^{-y^{2} / 2}}{\sqrt{2 \pi}}\left(1-F_{(0,1)}(y)\right)^{k-1} \\
& \quad \times F_{(0,1)}^{N-k}(y) d y+E\left[D_{i j}\right] \frac{(k-1) !(N-k) !}{N !} .
\end{aligned}
$$

The probability of the nonempty buffer for any user, $\alpha$, in terms of average throughput and traffic rate, is given as follows:

$$
\alpha=\frac{\lambda}{E\left[R_{i}\right]},
$$

where $\lambda$ is the average arrival traffic rate per user. By substituting (20) into (19), $E\left[R_{i}\right]$ becomes

$$
\begin{aligned}
E\left[R_{i}\right]= & \sum_{k=1}^{N} \frac{\lambda}{E\left[R_{i}\right]}\left(1-\frac{\lambda}{E\left[R_{i}\right]}\right)^{k-1} \\
& \times \sum_{j=1}^{M} \sigma_{D_{i j}} \int_{-\infty}^{\infty} y \frac{e^{-y^{2} / 2}}{\sqrt{2 \pi}}\left(1-F_{(0,1)}(y)\right)^{k-1} F_{(0,1)}^{N-k}(y) d y \\
& +E\left[D_{i j}\right] \frac{(k-1) !(N-k) !}{N !} .
\end{aligned}
$$


As $E\left[R_{i}\right]$ represents the throughput of user $i$ in the system, the average throughput of the entire system is

$$
E[R]=\sum_{i=1}^{N} E\left[R_{i}\right]
$$

4.2. Fairness Index. Jain's fairness index is a well-known quantitative metric that is widely used in wireless communications to measure fairness, and it is defined as follows [14]:

$$
J\left(x_{1}, x_{2}, x_{3}, \ldots, x_{N}\right)=\frac{\left(\sum_{i=1}^{N} x_{i}\right)^{2}}{N \sum_{i=1}^{N} x_{i}^{2}},
$$

where $x_{i}$ is the amount of resources accessed by user $i$ among $N$ competing users. Based on the result for the average throughput for user $i$, as given in (21), it is straightforward to express the Jain's fairness index of the users' throughput as follows:

$$
J\left(E\left[R_{1}\right], E\left[R_{2}\right], E\left[R_{3}\right], \ldots, E\left[R_{N}\right]\right)=\frac{\left(\sum_{i=1}^{N} E\left[R_{i}\right]\right)^{2}}{\sum_{i=1}^{N} E\left[R_{i}\right]^{2}} .
$$

For nonbursty traffic (full-buffer scenario), the analysis is the same as for bursty traffic given above, except that $\alpha$ (the probability of having non-empty buffer) is equal to 1 .

4.3. Average Packet Delay. In order to calculate the packet delay, we model the system by using the M/G/1 queuing model. Hence, the average packet delay is given by

$$
\omega_{i}=\frac{1}{E\left[R_{i}\right]}+\frac{\lambda_{i}\left(1 /\left(E^{2}\left[R_{i}\right]+\sigma_{R_{i}}^{2}\right)\right)}{2\left(1-\left(\lambda_{i} / E\left[R_{i}\right]\right)\right)},
$$

where $\sigma_{R_{i}}^{2}$ is the throughput variance. In order to determine $\sigma_{R_{i}}^{2}$, we calculate $E\left[R_{i}^{2}(n)\right]$ using (3) as follows:

$$
\begin{aligned}
E\left[R_{i}^{2}(n)\right]= & \left(\frac{T_{c}-1}{T_{c}}\right)^{2} E\left[R_{i}^{2}(n-1)\right] \\
& +\frac{1}{T_{c}^{2}} \sum_{i=1}^{N} \alpha^{2}(1-\alpha)^{2(i-1)} E\left[\sum_{j=1}^{M} D_{i j}(n) I_{i j}^{i}(n)\right]^{2} \\
& +\frac{2\left(T_{c}-1\right)}{T_{c}^{2}} E\left[R_{i}(n-1)\right] \\
& \times E\left[\sum_{i=1}^{N} \alpha(1-\alpha)^{i-1} \sum_{j=1}^{M} D_{i j}(n) I_{i j}^{i}(n)\right] .
\end{aligned}
$$

By assuming stationary throughput per user, we can use $E\left[R_{i}(n)\right]=E\left[R_{i}(n-1)\right]$. Therefore, $(26)$ can be re-written as follows:

$$
\begin{aligned}
\left(2 T_{c}-1\right) E\left[R_{i}^{2}\right]= & \sum_{i=1}^{N} \alpha^{2}(1-\alpha)^{2(i-1)} E \\
& \times\left[\sum_{j=1}^{M} D_{i j}(n) I_{i j}^{i}(n)\right]^{2}+2\left(T_{c}-1\right) \\
& \times E\left[R_{i}\right] E\left[\sum_{i=1}^{N} \alpha(1-\alpha)^{i-1} \sum_{j=1}^{M} D_{i j}(n) I_{i j}^{i}(n)\right] .
\end{aligned}
$$

In order to determine $E\left[R_{i}^{2}\right]$, we need to find $E\left[\sum_{j=1}^{M} D_{i j}(n) I_{i j}^{i}(n)\right]^{2}$, which can be expressed as follows:

$$
E\left[\sum_{j=1}^{M} D_{i j}(n) I_{i j}^{i}(n)\right]^{2}=\sum_{j=1}^{M} E\left(D_{i j}^{2} I_{i j}^{i}\right)+\sum_{j=1}^{M} \sum_{\substack{h=1, h \neq j}}^{M} E\left(D_{i j} D_{i h} I_{i j}^{i} I_{i h}^{i}\right),
$$

and then can be re-written as

$$
\begin{aligned}
& E\left[\sum_{j=1}^{M} D_{i j}(n) I_{i j}^{i}(n)\right]^{2} \\
& =\sum_{j=1}^{M} \operatorname{Pr}\left(I_{i j}^{i}=1\right) \\
& \quad \times \int_{-\infty}^{\infty} x^{2} f_{D_{i j}}\left(x \mid I_{i j}^{i}=1\right) d x \\
& \quad+\sum_{j=1}^{M} \operatorname{Pr}\left(I_{i j}^{i}=1\right)\left(\int_{-\infty}^{\infty} x f_{D_{i j}}\left(x \mid I_{i j}^{i}=1\right) d x\right) \\
& \quad \times \sum_{\substack{h=1 \\
h \neq j}}^{M} \operatorname{Pr}\left(I_{i h}^{i}=1\right)\left(\int_{-\infty}^{\infty} x f_{D_{i h}}\left(x \mid I_{i h}^{i}=1\right) d x\right) .
\end{aligned}
$$

The first term in the right-hand side of (29) can be further written as follows:

$$
\begin{aligned}
& \sum_{j=1}^{M} \operatorname{Pr}\left(I_{i j}^{i}=1\right) \int_{-\infty}^{\infty} x^{2} f_{D_{i j}}\left(x \mid I_{i j}^{i}=1\right) d x \\
& \quad=\sum_{j=1}^{M} \int_{-\infty}^{\infty} x^{2} f_{D_{i j}}(x) \operatorname{Pr}\left(I_{i j}^{i}=1 \mid D_{i j}=x\right) d x .
\end{aligned}
$$


Using (9) and the assumption of stationary first-order ergodic $R_{i}$ [9], (30) becomes

$$
\begin{aligned}
\sum_{j=1}^{M} \operatorname{Pr}\left(I_{i j}^{i}=1\right) \int_{-\infty}^{\infty} x^{2} f_{D_{i j}}\left(x \mid I_{i j}^{i}=1\right) d x \\
=\sum_{j=1}^{M} \int_{-\infty}^{\infty} x^{2} f_{D_{i j}}(x)\left(1-F_{D_{i j}}\left(\frac{E\left[R_{l}(n)\right]}{E\left[R_{i}(n)\right]} x\right)\right)^{i-1} \\
\times \prod_{\substack{l=1, l \neq i}}^{N-i} F_{D_{i j}}\left(\frac{E\left[R_{l}(n)\right]}{E\left[R_{i}(n)\right]} x\right) d x
\end{aligned}
$$

which can be simplified to

$$
\begin{gathered}
\sum_{j=1}^{M} \operatorname{Pr}\left(I_{i j}^{i}=1\right) \int_{-\infty}^{\infty} x^{2} f_{D_{i j}}\left(x \mid I_{i j}^{i}=1\right) d x \\
=\sum_{j=1}^{M} \int_{-\infty}^{\infty}\left(y \sigma_{D_{i j}}+E\left[D_{i j}\right]\right)^{2} f_{D_{i j}}(y) \\
\times\left(1-F_{(0,1)}(y)\right)^{i-1} F_{(0,1)}^{N-i}(y) d x .
\end{gathered}
$$

Then, by simply expressing $\left(y \sigma_{D_{i j}}+E\left[D_{i j}\right]\right)^{2}$, (32) can be re-written as follows:

$$
\begin{aligned}
& \sum_{j=1}^{M} \operatorname{Pr}\left(I_{i j}^{i}=1\right) \int_{-\infty}^{\infty} x^{2} f_{D_{i j}}\left(x \mid I_{i j}^{i}=1\right) d x \\
& =\sum_{j=1}^{M} \sigma_{D_{i j}}^{2} \int_{-\infty}^{\infty} y^{2} f_{D_{i j}}(y)\left(1-F_{(0,1)}(y)\right)^{i-1} F_{(0,1)}^{N-i}(y) d x \\
& \quad+2 \sigma_{D_{i j}} E\left[D_{i j}\right] \int_{-\infty}^{\infty} y f_{D_{i j}}(y)\left(1-F_{(0,1)}(y)\right)^{i-1} F_{(0,1)}^{N-i}(y) d x \\
& \quad+E\left[D_{i j}\right]^{2} \int_{-\infty}^{1} y^{2} f_{D_{i j}}(y)\left(1-F_{(0,1)}(y)\right)^{i-1} F_{(0,1)}^{N-i}(y) d x .
\end{aligned}
$$

Thus, $\sum_{j=1}^{M} \operatorname{Pr}\left(I_{i j}^{i}=1\right) \int_{-\infty}^{\infty} x^{2} f_{D_{i j}}\left(x \mid I_{i j}^{i}=1\right) d x$ can be expressed as follows:

$$
\begin{aligned}
\sum_{j=1}^{M} \operatorname{Pr}\left(I_{i j}^{i}=1\right) \int_{-\infty}^{\infty} x^{2} f_{D_{i j}}\left(x \mid I_{i j}^{i}=1\right) d x \\
=\sum_{j=1}^{M} \sigma_{D_{i j}}^{2} \int_{-\infty}^{\infty} y^{2} f_{D_{i j}}(y)\left(1-F_{(0,1)}(y)\right)^{i-1} F_{(0,1)}^{N-i}(y) d x \\
+2 \sigma_{D_{i j}} E\left[D_{i j}\right] \int_{-\infty}^{\infty} y f_{D_{i j}}(y)\left(1-F_{(0,1)}(y)\right)^{i-1} F_{(0,1)}^{N-i}(y) d x \\
+\frac{(i-1) E\left[D_{i j}\right]}{N V(N-i) !} .
\end{aligned}
$$

Next, we determine the second term in the right-hand side of (29), which can be re-written as follows:

$$
\begin{aligned}
& \sum_{j=1}^{M} \operatorname{Pr}\left(I_{i j}^{i}=1\right)\left(\int_{-\infty}^{\infty} x f_{D_{i j}}\left(x \mid I_{i j}^{i}=1\right) d x\right) \\
& \quad \times \sum_{\substack{h=1 \\
h \neq j}}^{M} \operatorname{Pr}\left(I_{i k}^{i}=1\right)\left(\int_{-\infty}^{\infty} x f_{D_{i h}}\left(x \mid I_{i h}^{i}=1\right) d x\right) \\
& =\sum_{j=1}^{M}\left(\sigma_{D_{i j}} \int_{-\infty}^{\infty} \frac{y e^{-y^{2} / 2}}{\sqrt{2 \pi}}\left(1-F_{(0,1)}(y)\right)^{i-1} F_{(0,1)}(y)^{N-i} d y\right. \\
& \left.\quad+\frac{(i-1) E\left[D_{i j}\right]}{N ! /(N-i) !}\right) \\
& \times \sum_{\substack{h=1, h \neq j}}^{M}\left(\sigma_{D_{i k}} \int_{-\infty}^{\infty} \frac{y e^{-y^{2} / 2}}{\sqrt{2 \pi}}\left(1-F_{(0,1)}(y)\right)^{i-1}\right. \\
& \left.\times F_{(0,1)}(y)^{N-i} d y+\frac{(i-1) E\left[D_{i h}\right]}{N ! /(N-i) !}\right) .
\end{aligned}
$$

From (29), (34) and (35), $E\left[\sum_{j=1}^{M} D_{i j}(n) I_{i j}^{i}(n)\right]^{2}$ can be expressed as follows:

$$
\begin{aligned}
& E\left[\sum_{j=1}^{M} D_{i j}(n) I_{i j}^{i}(n)\right]^{2} \\
& =\sum_{j=1}^{M} \sigma_{D_{i j}}^{2} \int_{-\infty}^{\infty} y^{2} f_{D_{i j}}(y)\left(1-F_{(0,1)}(y)\right)^{i-1} F_{(0,1)}^{N-i}(y) d x \\
& +2 \sigma_{D_{i j}} E\left[D_{i j}\right] \int_{-\infty}^{\infty} y f_{D_{i j}}(y)\left(1-F_{(0,1)}(y)\right)^{i-1} F_{(0,1)}^{N-i}(y) d x \\
& +\frac{(i-1) E\left[D_{i j}\right]}{N ! /(N-i) !} \\
& +\sum_{j=1}^{M}\left(\sigma_{D_{i j}} \int_{-\infty}^{\infty} \frac{y e^{-y^{2} / 2}}{\sqrt{2 \pi}}\left(1-F_{(0,1)}(y)\right)^{i-1} F_{(0,1)}(y)^{N-i} d y\right. \\
& \left.\quad+\frac{(i-1) E\left[D_{i j}\right]}{N ! /(N-i) !}\right) \\
& \quad \times \sum_{h=1,}^{M}\left(\sigma_{D_{i k}} \int_{-\infty}^{\infty} \frac{y e^{-y^{2} / 2}}{\sqrt{2 \pi}}\left(1-F_{(0,1)}(y)\right)^{i-1} F_{(0,1)}(y)^{N-i} d y\right. \\
& h \neq j
\end{aligned}
$$


Then, we simplify the second term in the right-hand side of (27) as follows:

$$
\begin{gathered}
2\left(T_{c}-1\right) E\left[R_{i}\right] E\left[\sum_{i=1}^{N} \alpha(1-\alpha)^{i-1} \sum_{j=1}^{M} D_{i j}(n) I_{i j}^{i}(n)\right] \\
=2\left(T_{c}-1\right) E\left[R_{i}\right] E\left[R_{i}\right]=2\left(T_{c}-1\right) E\left[R_{i}\right]^{2} .
\end{gathered}
$$

Substituting (36) and (37) in (27), it can be easily shown that the throughput variance is expressed as:

$$
\begin{aligned}
& \sigma_{R_{i}}^{2}=E\left[R_{i}^{2}\right]-E\left[R_{i}\right]^{2} \\
& =\frac{1}{2\left(T_{c}-1\right)}\left(\sum_{i=1}^{N} \alpha^{2}(1-\alpha)^{2(i-1)}\right. \\
& \times \sum_{j=1}^{M} \sigma_{D_{i j}}^{2} \int_{-\infty}^{\infty} y^{2} f_{D_{i j}}(y)\left(1-F_{(0,1)}(y)\right)^{i-1} F_{(0,1)}^{N-i}(y) d x \\
& +2 \sigma_{D_{i j}} E\left[D_{i j}\right] \int_{-\infty}^{\infty} y f_{D_{i j}}(y)\left(1-F_{(0,1)}(y)\right)^{i-1} F_{(0,1)}^{N-i}(y) d y \\
& +\frac{(i-1) E\left[D_{i j}\right]}{N ! /(N-i) !} \sum_{i=1}^{N} \alpha^{2}(1-\alpha)^{2(i-1)} \\
& \times \sum_{j=1}^{M}\left(\sigma_{D_{i j}} \int_{-\infty}^{\infty} \frac{y e^{-y^{2} / 2}}{\sqrt{2 \pi}}\left(1-F_{(0,1)}(y)\right)^{i-1} F_{(0,1)}^{N-i}(y) d y\right. \\
& +\frac{(i-1) E\left[D_{i j}\right]}{N ! /(N-i) !} \\
& \times \sum_{\substack{k=1, k \neq j}}^{M}\left(\sigma_{D_{i k}} \int_{-\infty}^{\infty} \frac{y e^{-y^{2} / 2}}{\sqrt{2 \pi}}\left(1-F_{(0,1)}(y)\right)^{i-1}\right. \\
& \left.\left.\times F_{(0,1)}^{N-i}(y) d y+\frac{(i-1) E\left[D_{i k}\right]}{N ! /(N-i) !}\right)\right) .
\end{aligned}
$$

By substituting (21) and (38) in (25), we can calculate the average packet delay $\left(\omega_{i}\right)$.

\section{Numerical and Simulation Results}

The accuracy of the analytical closed-form expressions for the average throughput, fairness index, and packet delay (derived in Section 4) is examined by comparing the analytical results with simulation results. Computer simulations of one cell with $N$ users are conducted independently of the analytical expressions derived in the previous section to estimate the average throughput, fairness index, and packet delay. We set the signal bandwidth to $20 \mathrm{MHz}$, the carrier frequency to $2 \mathrm{GHz}$, the noise power to $-130 \mathrm{dBW}$, and $T_{c}$ to 5000 frames (except in Figures 2, 3, and 10). In addition, we consider a path loss exponent of 4 , the standard deviation of the lognormal shadowing equal to $10 \mathrm{~dB}$, the cell radius set to $1500 \mathrm{~m}$, the number of users, $N$, in the cell equal to

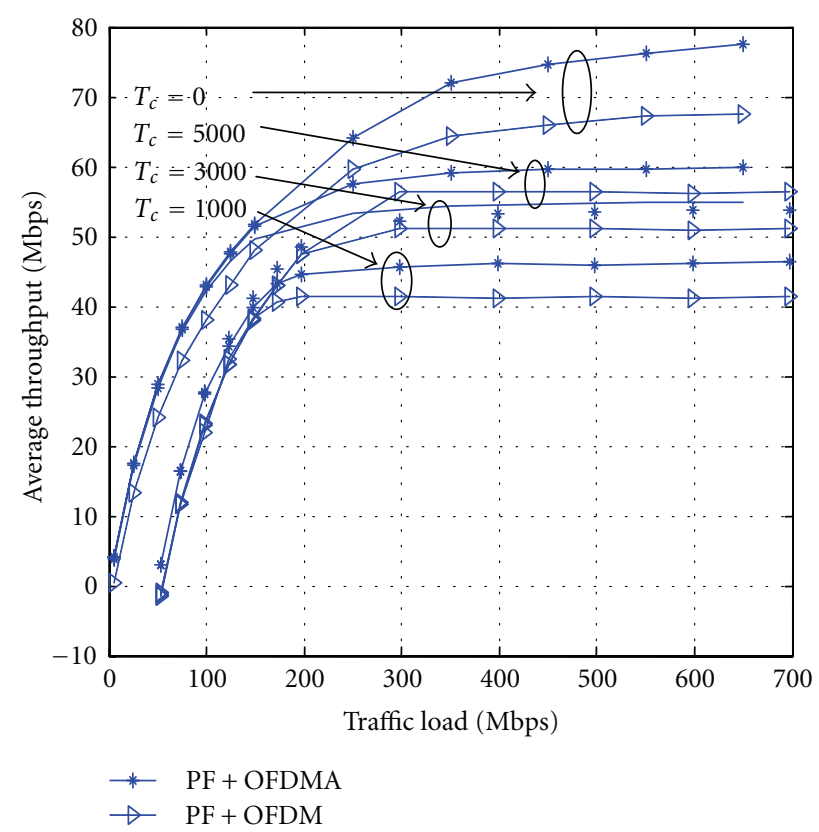

FIgure 2: Average throughput of the PF with OFDMA and PF with OFDM.

32, the frame duration of $2 \mathrm{~ms}$, and the packet size of 180 bits. The number of subbands, $M$, is 32 and the number of subcarriers, $S$, is 256 . We use Poisson traffic with an arrival rate of $\lambda$, which is kept as a variable to control the traffic load given by $\lambda N$.

We first analyze the effect of the averaging window $\left(T_{c}\right)$ and the impact of using OFDMA instead of OFDM. In OFDM, all subcarriers are given to the selected user by the PF. As shown in Figure 2 (when $T_{c}>0$ ) the larger the $T_{c}$ the higher the throughput. When $T_{c}$ increases, PF needs more time to compensate disadvantaged users (with low SNR), which leads to a higher throughput for the advantaged users (with good SNR). As a result, the average throughput increases. On the other hand, when $T_{c}=0, \mathrm{PF}$ losses its fairness and becomes an opportunistic scheduling algorithm which favors advantaged users, and it is known that opportunistic scheduling algorithms achieve the highest average throughput (but at the expense of the fairness). Also, it is evident from Figure 2 that PF with OFDMA has higher throughput than that of PF with OFDM, as the former efficiently utilizes the resources in the frequency domain, and can handle efficiently the bursty traffic because of the subband sharing.

The Jain's fairness index of PF with OFDMA and PF with OFDM is depicted in Figure 3. Both algorithms show approximately the same values of Jain's fairness index with a slight improvement for PF with OFDMA. Also, we can notice that as $T_{c}$, increases (when $T_{c}>0$ ), the fairness index decreases, as the algorithm becomes less fair (as discussed above). Furthermore, the lowest Jain's fairness index is associated with $T_{c}=0$ because this is the case when PF becomes completely opportunistic, as discussed above. 

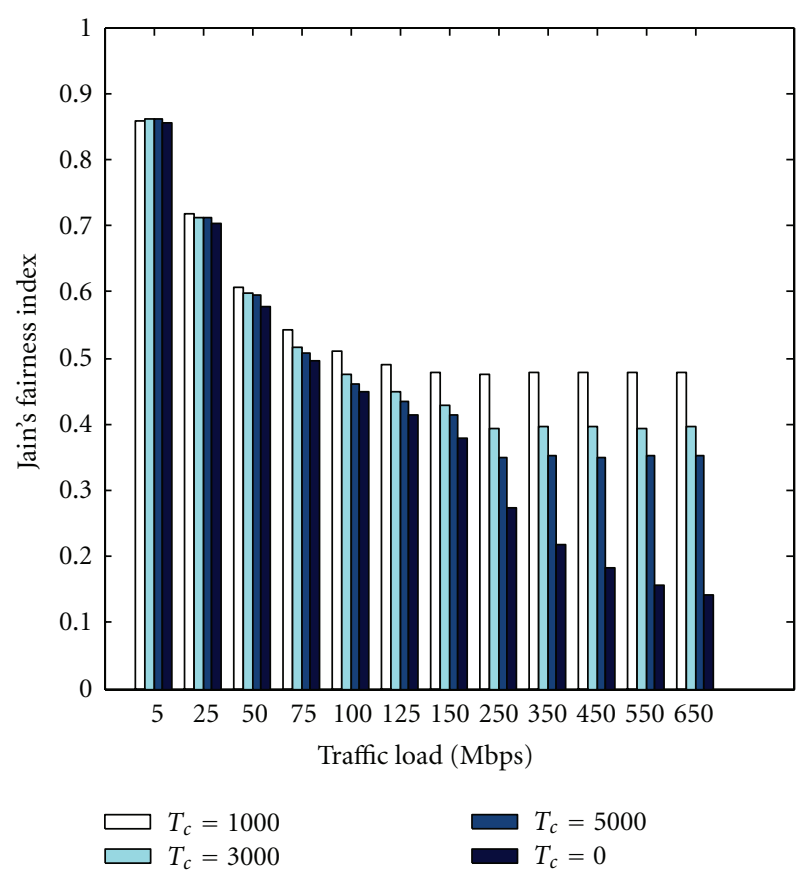

(a)

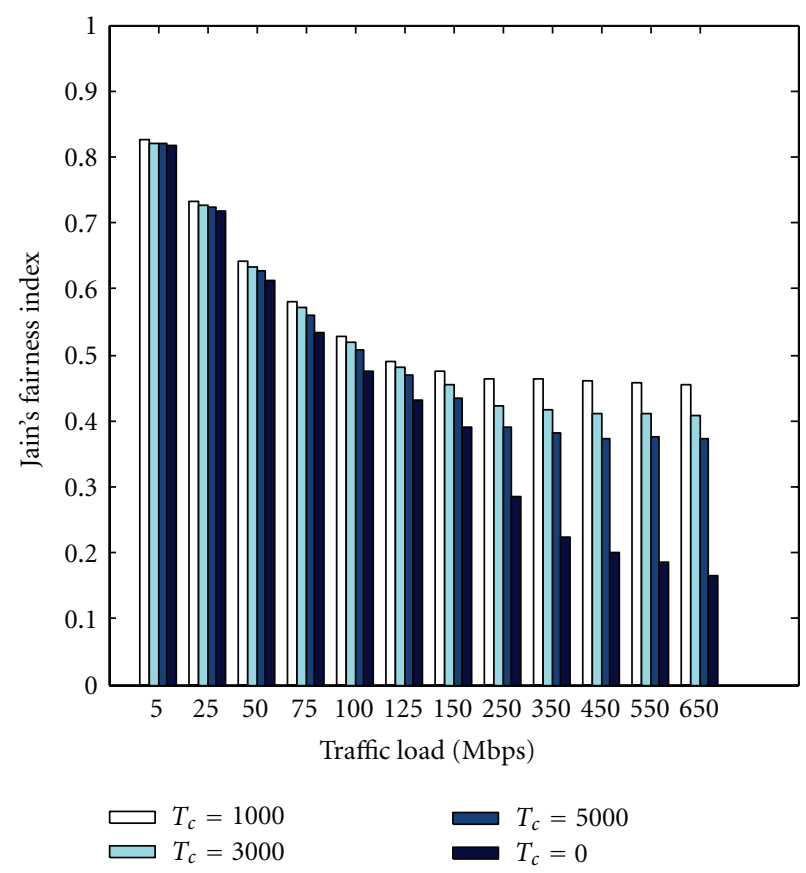

(b)

FIGURE 3: Fairness index of the PF scheduling with (a) OFDMA (b) OFDM.

In Figures 4 and 5, the throughput and the Jain's fairness index of the system are, respectively, shown versus the total traffic load in the cell. Results obtained from both analytical expressions in (20) and (21) and simulations are presented. It is noteworthy the good agreement between these results, which validate our analytical solution. From

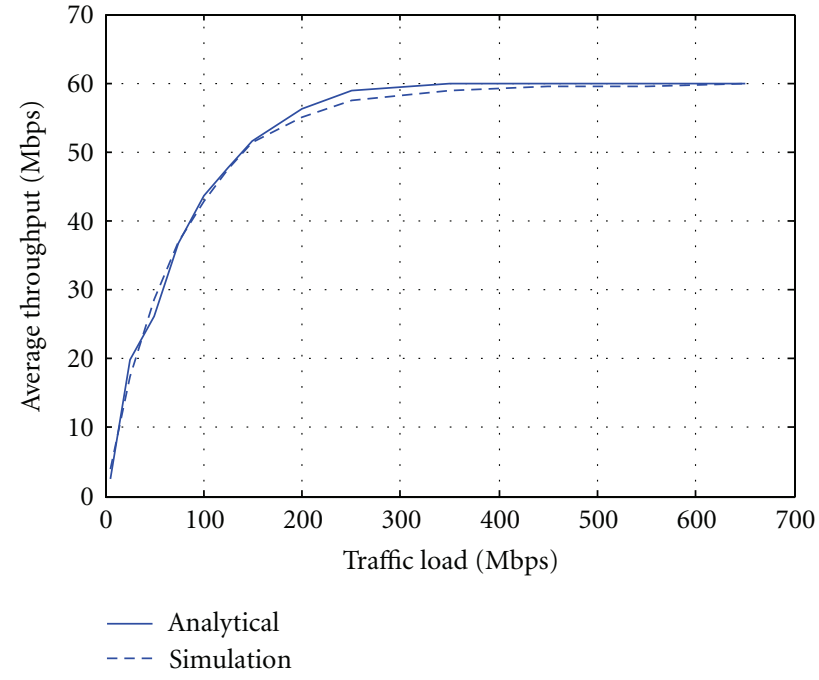

Figure 4: Average throughput versus traffic load.

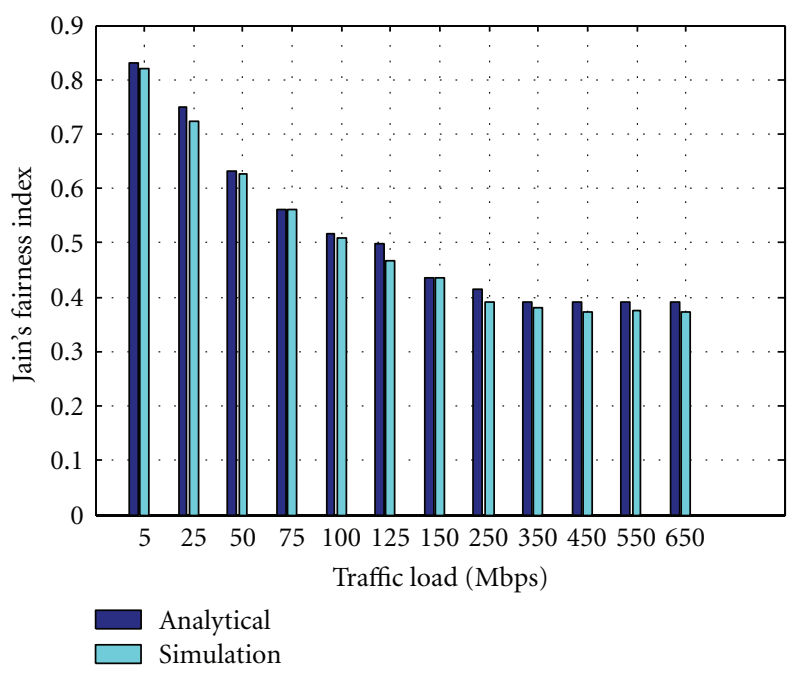

FIGURE 5: Jain's fairness index versus traffic load.

Figure 4, one can observe that (as expected) the average throughput increases sharply at low traffic load, and then it saturates at high traffic load. On the other hand, as shown in Figure 5, the fairness index decreases with the traffic load increase, and it saturates at high traffic load. This is because as the traffic load increases, fewer resources become available and it becomes more difficult to satisfy the demand of all users.

The performance of the PF scheduling algorithm that we propose in [8] and the agreement between analytical and simulation results are also investigated for a different number of users, $N$, where the traffic load expected from each user is assumed to be $10 \mathrm{Mbps}$ and the averaging window, $T_{c}$, for the simulation, is selected to be 5000 . Figures 6 and 7 show the average throughput and Jain's fairness index versus the number of users, respectively. Again, it is straightforward to notice that there is good matching between analytical and simulation results. From Figure 6, one can see the increase in 


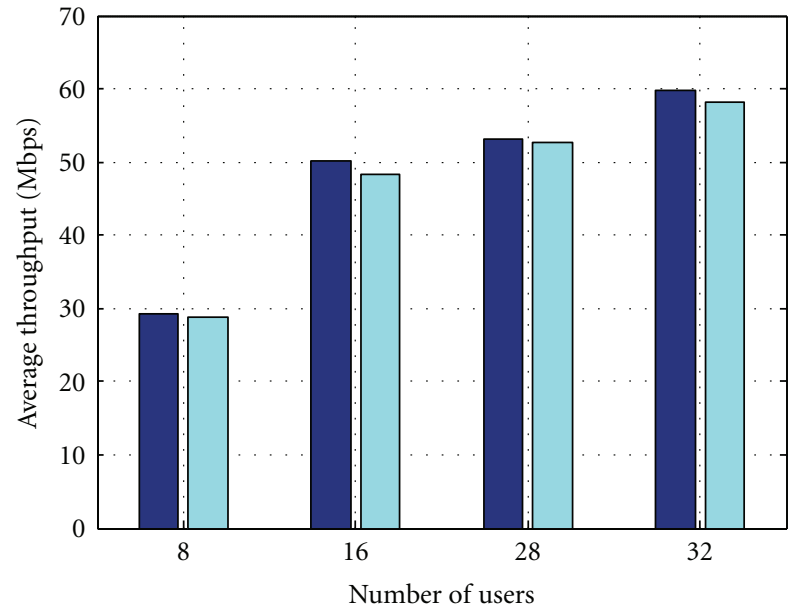

Analytical

Simulation

FIGURE 6: Average throughput versus number of users.

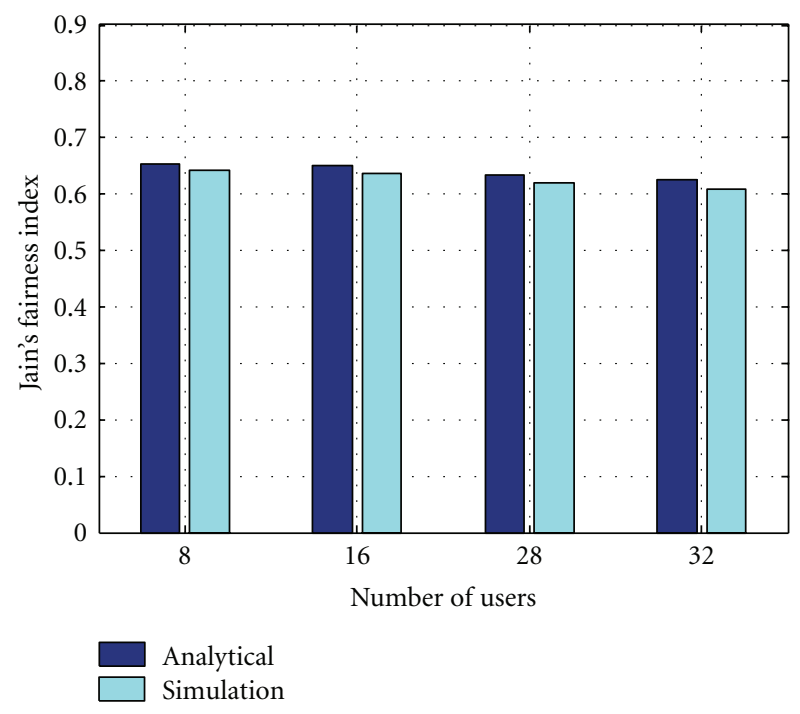

FiguRE 7: Jain's fairness index versus number of users.

the average throughput when the number of users increases for both analytical and simulation bars. This can be easily explained as follows: as the number of users increases, the traffic loads increase in the system. Also, as the number of users increases, the chance of scheduling users on subbands with preferable channel gain increases, so the scheduling algorithm utilizes the multiuser diversity. From Figure 7, we notice a slight fairness index decrease when the number of users increases. This fairness index decrease is expected, as the competition when the number of users increases.

Figure 8 shows the throughput performance at different number of subbands $(M)$. The available frequency bandwidth is divided into different number of subbands to study the behavior of the system with different numbers of subbands. It is evident that the analytical results and the simulation results agree very well. We also notice that

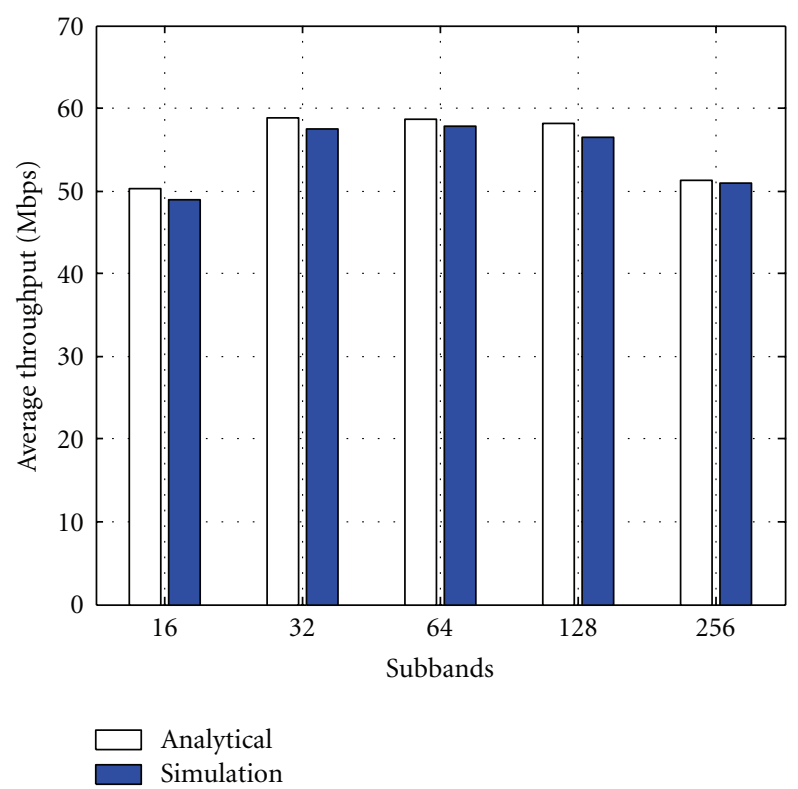

FIGURE 8: Throughput versus the number of subbands (traffic load $=250 \mathrm{Mbps}$ ).

the throughput reaches the maximum when the number of subbands equals 64 . When the number of subbands is small, the number of subcarriers per subband is larger. Hence, the use of the adaptive coding and modulation for all the subcarriers, based on the subcarriers with worst channel conditions, will waste the resources of many subcarriers with favorable channel conditions. On the other hand, when the number of subbands is large, few subcarriers are grouped to create a subband, which degrades the throughput because of the increasing amount of unused fractions of subbands at the end of time frames. In other words, when the number of subbands increases, the number of subbands that are not fully utilized at the end of time frames increases, which degrades the throughput performance.

Figure 9 shows the Jain's fairness index at different number of subbands. We notice that the number of subbands does not affect the fairness of the system, as all users suffer from the same degradation of subbands utilization. Thus, the chance of accessing the resources will be affected equally for all users in the system, which keeps the fairness performance the same, regardless of the number of subbands.

Figure 10 shows the packet delay versus traffic load for the proposed scheduling algorithm, for $T_{c}$ equals 5000, 3000, and 1000. It is evident that as the traffic load increases, the competition between users becomes harder, which causes more packets to wait longer time in the users queues. Also, we notice that when $T_{c}$ increases, the packet delay increases. This can be explained as follows. When $T_{c}$ increases, the scheduler tries to maximize the system throughput by forcing greedy treatment among users by allocating most of the resources to a few of users who have favorable channel conditions. That behavior blocks more packets for requesting users, which increases the average packet delay in the system. 


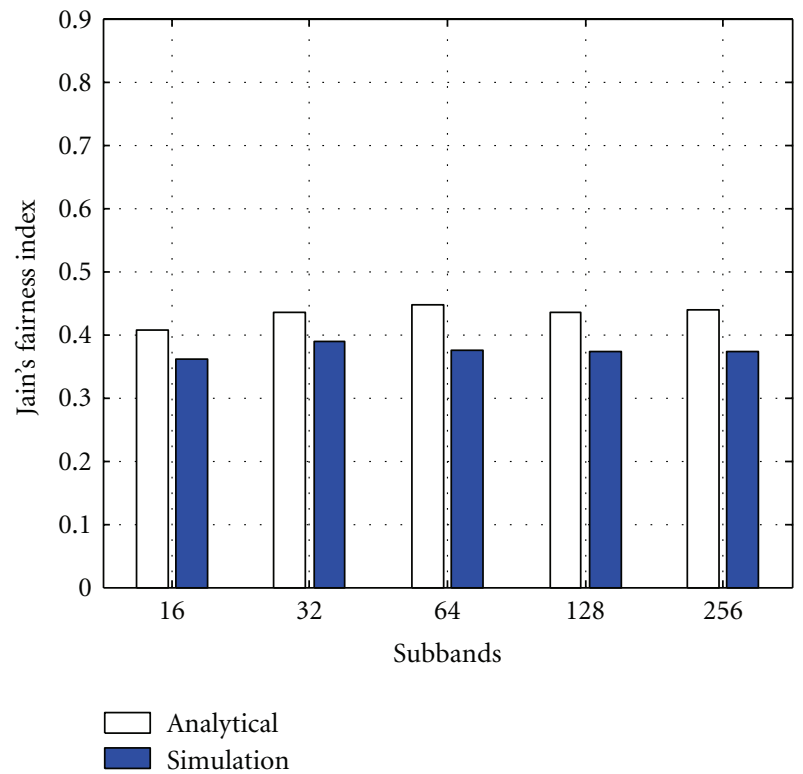

Figure 9: Jain's fairness index versus the number of subbands (traffic load $=250 \mathrm{Mbps}$ ).

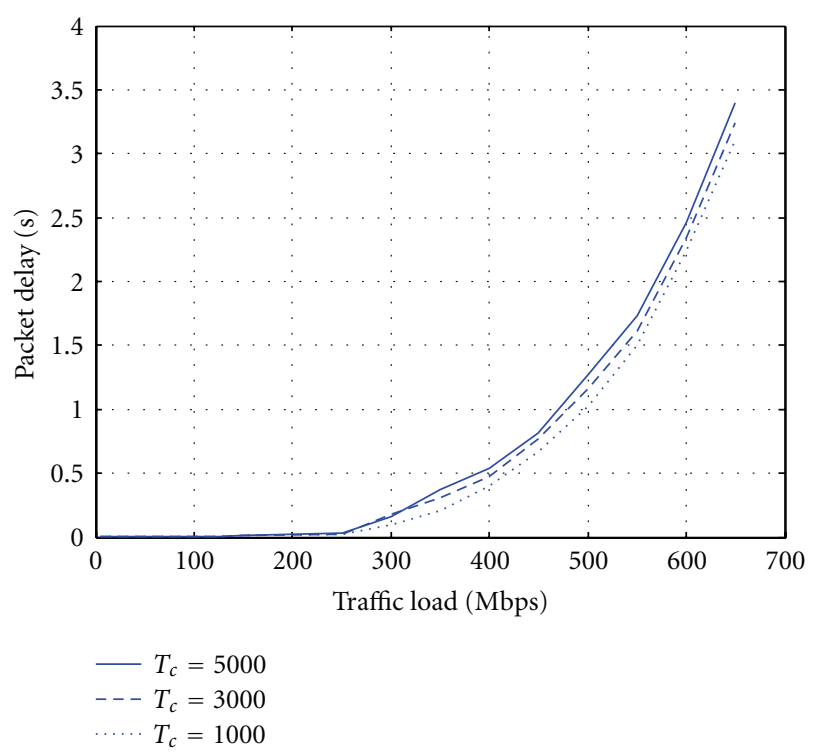

Figure 10: Mean packet delay versus traffic load of the proposed algorithm.

Figure 11 shows the packet delay versus traffic load for the proposed scheduling algorithm (PF with OFDMA), analytically and by simulation, and the packet delay for the $\mathrm{PF}$ with OFDM, where the observation window $T_{c}$ equals 5000 . As we notice, the analytical curve agrees very well with the simulation curve. Also, we notice a slight improvement of the proposed scheduling algorithm over the PF with OFDM. We notice that on high traffic load $(650 \mathrm{Mbps})$ our proposed scheduling algorithm mean packet delay equals 3.75 seconds while the mean packet delay of PF with OFDM equals 3.45 seconds.

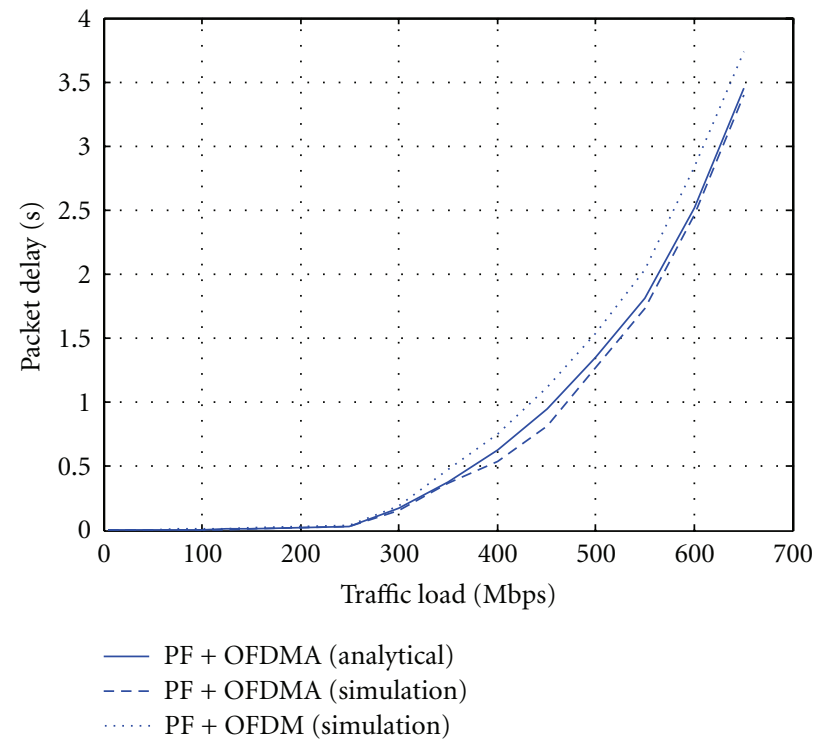

FIGURE 11: Mean packet delay versus traffic load for the proposed algorithm analytically and by simulation for PF with OFDMA and PF with OFDM.

It is noteworthy that there is a small difference between the analytical and simulation results. This result difference can be explained because of the approximations that have been introduced while deriving the analytical model. Such approximations simplify the model at the cost of minor result deviations.

\section{Conclusion}

In this work, the PF scheduling is investigated for OFDMA wireless systems. The main contribution of this work is the analytical evaluation of the performance of PF scheduling algorithm in OFDMA systems. We derive approximate closed-form expressions for the average throughput, Jain's fairness index, and packet delay as the performance metrics. The algorithm performance is investigated for a broad range of the traffic load and number of subbands. We compare the performance of the proposed algorithm (PF with OFDMA) with that of PF with OFDM. In addition, we verify the correctness and accuracy of the analytical solution through simulations. Analytical and simulation results are in good agreement, which validates our analytical performance analysis. In future work, we plan to extend the analysis to the case of different probabilities of the non-empty buffer for different users. We will also consider other fading distributions, such as the Rician distribution.

\section{Acknowledgments}

The authors are grateful to the anonymous reviewers and the editor for their constructive comments that improved the quality of the paper. This work has been supported by the NSERC Discovery Grant Program. 


\section{References}

[1] L. C. Wang and W. J. Lin, "Throughput and fairness enhancement for OFDMA broadband wireless access systems using the maximum C/I scheduling," in Proceedings of the IEEE 60th Vehicular Technology Conference (VTC '04), pp. 4696-4700, September 2004.

[2] Y. Ben-Shimol, I. Kitroser, and Y. Dinitz, "Two-dimensional mapping for wireless OFDMA systems," IEEE Transactions on Broadcasting, vol. 52, no. 3, pp. 388-396, 2006.

[3] C. Y. Wong, R. S. Cheng, K. B. Letaief, and R. D. Murch, "Multiuser OFDM with adaptive subcarrier, bit, and power allocation," IEEE Journal on Selected Areas in Communications, vol. 17, no. 10, pp. 1747-1758, 1999.

[4] I. C. Wong and B. L. Evans, "Optimal resource allocation in the OFDMA downlink with imperfect channel knowledge," IEEE Transactions on Communications, vol. 57, no. 1, pp. 232-241, 2009.

[5] H. J. Zhu and R. H. M. Hafez, "Scheduling schemes for multimedia service in wireless OFDM systems," IEEE Wireless Communications, vol. 14, no. 5, pp. 99-105, 2007.

[6] N. Ruangchaijatupon and Y. Ji, "Simple proportional fairness scheduling for OFDMA frame-based wireless systems," in Proceedings of the IEEE Wireless Communications and Networking Conference (WCNC '08), pp. 1593-1597, usa, April 2008.

[7] K. W. Choi, W. S. Jeon, and D. G. Jeong, "Resource allocation in OFDMA wireless communications systems supporting multimedia services," IEEE/ACM Transactions on Networking, vol. 17, no. 3, pp. 926-935, 2009.

[8] R. Almatarneh, M. Ahmed, and O. Dobre, "Frequency-time scheduling algorithm for OFDMA systems," in Proceedings of the Canadian Conference on Electrical and Computer Engineering (CCECE '09), pp. 766-771, May 2009.

[9] E. Liu and K. K. Leung, "Proportional fair scheduling: analytical insight under Rayleigh fading environment," in Proceedings of the IEEE Wireless Communications and Networking Conference (WCNC'08), pp. 1883-1888, April 2008.

[10] B. Sklar, "Rayleigh fading channels in mobile digital communication systems Part I: characterization," IEEE Communications Magazine, vol. 35, no. 7, pp. 90-100, 1997.

[11] B. Sklar, "Rayleigh fading channels in mobile digital communication systems Part II: mitigation," IEEE Communications Magazine, vol. 35, no. 9, pp. 148-155, 1997.

[12] L. C. Tran, T. A. Wysocki, A. Mertins, and J. Seberry, "A generalized algorithm for the generation of correlated Rayleigh fading envelopes in wireless channels," Eurasip Journal on Wireless Communications and Networking, vol. 2005, no. 5, pp. 801-815, 2005.

[13] P. J. Smith and M. Shafi, "On a Gaussian approximation to the capacity of wireless MIMO systems," in Proceedings of the International Conference on Communications (ICC '02), pp. 406-410, May 2002.

[14] R. Jain, D. Chiu, and W. Hawe, "A quantitative measure of fairness and discrimination for resource allocation in shared computer systems," DEC Report DEC-TR-301, Digital Equipment Corporation, Littleton, Mass, USA, 1984. 

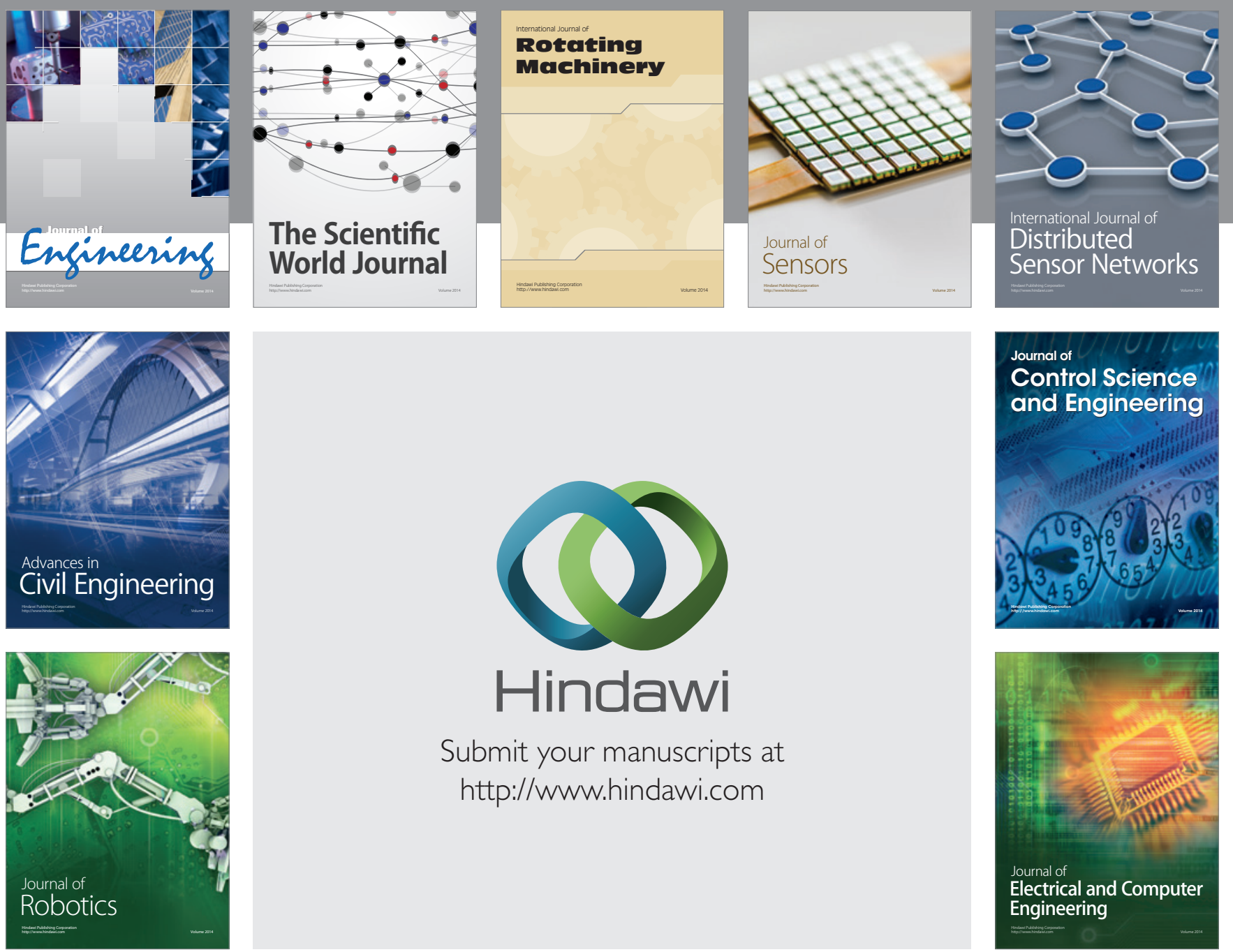

Submit your manuscripts at

http://www.hindawi.com
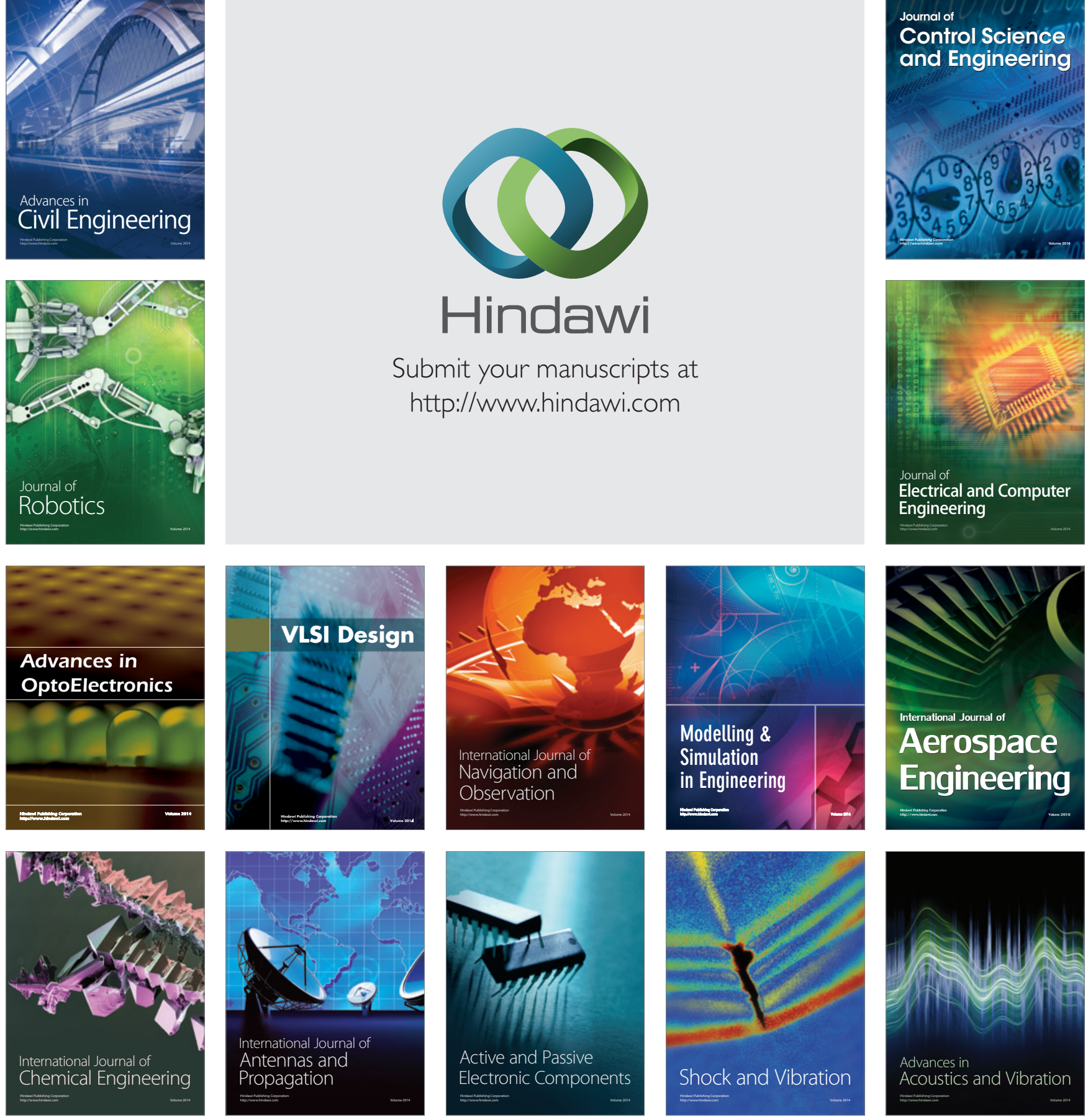